\title{
Advancing our knowledge of the complexity and management of intimate partner violence and co-occurring mental health and substance abuse problems in women Robin Mason* and Janice Du Mont
}

Address: Women's College Research Institute, Women's College Hospital, 790 Bay Street, $7^{\text {th }}$ floor, Toronto, ON M5G 1N8, Canada

*Corresponding author: Robin Mason (Robin.Mason@wchospital.ca)

Fl000Prime Reports 2015, 7:65 (doi:10.12703/P7-65)

All F1000Prime Reports articles are distributed under the terms of the Creative Commons Attribution-Non Commercial License (http://creativecommons.org/licenses/by-nc/3.0/legalcode), which permits non-commercial use, distribution, and reproduction in any medium, provided the original work is properly cited.

The electronic version of this article is the complete one and can be found at: http://fl000.com/prime/reports/m/7/65

\begin{abstract}
Globally, intimate partner violence (IPV) is a pervasive and insidious human rights problem with significant adverse physical health outcomes for women. Intimate partner violence has also been closely associated with poor mental health and substance use problems. However, little is known about the relationship among these co-occurring problems and how to best intervene or manage them. Here, we present findings from recent systematic reviews and meta-analyses (where available) to highlight developments in understanding and managing the complex co-occurring problems of intimate partner violence and mental health and substance use in women.
\end{abstract}

\section{Introduction}

There has been significant growth in our understanding of violence against women and its most common manifestation, IPV. According to the World Health Organization, IPV is defined as any behavior by an intimate partner or ex-partner that causes physical, sexual or psychological harm, including physical aggression, sexual coercion, psychological abuse and controlling behaviors $[1,2]$.

A simple PubMed search of the terms "domestic violence" (another, and the most common earlier term used to identify violence by an intimate partner) and "health" yielded 15,500 titles dating back to the 1960s. The titles of these early publications suggest that domestic violence was conceptualized primarily in terms of child maltreatment, abuse, and neglect. However, in 1975, several short papers on battered wives appeared in the journal Royal Society of Health. Sporadic publications that focused on women as victims appeared over the next two decades with a significant increase in research-related studies appearing in the mid-1990s. The intervening years have seen an accumulation of evidence on the pervasiveness and adverse health outcomes associated with violence against women in general, and IPV in particular, leading the Director-General of the World Health Organization to declare violence against women a "global health problem of epidemic proportions" [3].

Recent global estimates of violence against women reveal that approximately one in three ever-partnered women worldwide have experienced physical and/or sexual violence by an intimate partner at some point over the course of their lives [4]. Of these women, $42 \%$ sustain immediate physical injuries and $13 \%$ are fatally injured [5]. In fact, across studies, more than $38 \%$ of female homicide victims have been killed by an intimate partner [6]. There is also a known connection between IPV, HIV and other sexually transmitted infections (STIs) [7]. Women who have experienced IPV develop HIV and other STIs at rates 1.5 to 1.8 times more frequently than women who have not been abused [5]. Moreover, abused women are more likely to experience respiratory conditions, musculoskeletal conditions, cardiovascular disorders, diabetes, gastrointestinal symptoms, and report poorer functional physical health [8].

In addition, mental health problems such as depression, anxiety, post-traumatic stress disorder (PTSD), sleep 
disturbances, and suicidal ideation and attempts, as well as substance abuse problems, are also commonly associated with IPV $[9,10]$. For example, across 37 studies reviewed by Beydoun et al. [11], 11\% of women who experienced any type of IPV had a major depressive disorder and depressive symptoms were reported by $26 \%$ of victimized women, while findings from studies of women seeking substance abuse treatment report that nearly one in two had experienced IPV in the preceding year $[12,13]$. Smith and colleagues [14] examined abused women's use of alcohol as well as other substances using data from the American National Epidemiologic Survey on Alcohol and Related Conditions. They reported that among IPV victims, alcohol use disorders were most prevalent $(24.6 \%)$, followed by cannabis use disorders (7.4\%). Cocaine use disorders and opioid use disorders were less prevalent $(2.0 \%$ and $2.4 \%$, respectively). However, the risk of IPV victimization was greater for those with concurrent alcohol and cannabis use disorders than for those with either problem on its own.

It has been suggested that the relationship of IPV to poor mental health and substance use may be mediated by stress, in particular the continuous stress of living in an unpredictably violent or abusive environment [15]. Yet, conflicting evidence suggests that women with poorer mental health and/or substance use problems are more vulnerable to experiences of abuse due to their impaired cognition, judgment, memory, or ability to discern potentially threatening situations [16]. Clearly, the relationships are complex and to effectively address these problems we need to better understand them. Which comes first? Can causality be determined? Should interventions target the problems sequentially or in parallel? Examining the evidence from recent systematic reviews and meta-analyses (where available) for answers to these questions and the appropriate interventions will allow us to better understand and help the women who experience these co-occurring problems.

\section{Co-occurring IPV and mental health problems}

According to a recent synthesis of the evidence worldwide, women who have experienced IPV are two to three times as likely to experience depression [11] and almost four times as likely to attempt suicide as non-abused women [5]. Despite the many serious and frequent physical problems resulting from IPV, Dillon et al. [8] found that 66 of the 75 studies they reviewed focused on poor mental health outcomes for women experiencing IPV. Among the 58 studies of mental health outcomes of IPV reviewed by Lagdon, Armour and Stringer [10], depression was the most common outcome, reported in 39 studies and by those who had experienced physical assaults as well as those who experienced psychological violence without physical assault. PTSD was a significant mental health outcome in 33 of these studies. Eighteen studies examined depression that co-occurred with other mental health problems; half of these reported co-occurring PTSD and the remainder noted suicidality, anxiety, sleep disturbance, and drug abuse. However, most of the studies included in both the Dillon and Lagdon reviews were cross-sectional and as Dillon and colleagues noted, "causal implications cannot be made from any of the studies examined, although some temporal associations have been found" ([8] p. 11).

Trevillion and colleagues [17] conducted a systematic review and meta-analysis to determine whether there are differences in prevalence of IPV by type of mental health disorder. They found that there was a high prevalence and increased odds of experiencing IPV for those with depression, anxiety or PTSD. Although they suggested in their review that the experience of mental health problems appeared to precede IPV, similar to the Dillon and Lagdon reviews, there were too few longitudinal studies to positively conclude a causal relationship.

A recent systematic review and meta-analysis of longitudinal studies examining IPV and depressive symptoms including suicide attempts was conducted by Devries and colleagues [18] in an attempt to determine the causal relationship of IPV to these mental health problems. Sixteen studies with 36,163 participants were analyzed. The authors summarized their findings by stating: "Our review provides evidence that experience of IPV increases the odds of incident depressive symptoms and of suicide attempts among women. We also found evidence that depressive symptoms can increase the odds of incident IPV in women" [19]. These authors concluded, as have other experts, that the relationship of IPV to mental health problems is complex and bi-directional.

Deciphering the causal relationship between IPV and mental health problems, including depression, anxiety, PTSD, and eating disorders, is challenging due to a number of issues, including the small number of available longitudinal studies, variability among types of abuse experienced, the frequency and severity of abuse experienced, confounders based on adverse childhood experiences, differing measures for assessing mental health, the recency of mental health problems, as well as the frequent failure of the included studies to control for the abuse of alcohol or other substances [17-20].

\section{Co-occurring IPV and substance use problems}

A number of cross-sectional and longitudinal studies have shown a strong association between IPV and substance use/misuse [21]. Gilbert and colleagues [22] attempted to 
establish the temporal relationships between different substances and types of IPV in a longitudinal study of abused women accessing hospital emergency departments. Their findings are suggestive of the complex relationships among specific substances and types of abuse experienced. For example, women who used heroin, crack or cocaine in the prior 6 months were twice as likely to experience later IPV and almost three times as likely to report physical injury as non-using women. Sexual IPV was significantly associated with subsequent use of crack or cocaine. No significant associations were found for alcohol use and subsequent IPV but marginal associations were found for experiencing IPV and subsequent binge drinking. Afifi and colleagues [23] examined substance use, IPV victimization and perpetration among the general population and found that IPV victimization increased the odds of any type of substance use disorder except sedatives, with particularly strong associations shown for alcohol use and cannabis use.

The most robust research to date on IPV and substance use problems has focused on alcohol, a recognized risk factor for the perpetration of violence [23-25]. Alcohol use by abused women was explored in a recent global synthesis of the literature examining IPV prevalence and sequelae. The authors of the report noted that the best study they reviewed reported that women who had been abused by a partner were almost twice as likely as non-abused women to have an alcohol use disorder [5]. Some research has suggested that women's use of alcohol and other substances precedes experiences of IPV, while others have reported that substance abuse increased subsequent to IPV [5]. However, similar to those studies that have examined the relationship of IPV to mental health problems, most have been cross-sectional in design.

A recent systematic review attempted to disentangle the causal relationship of IPV to alcohol use among abused women but was only able to reiterate their bi-directional nature. The authors, who examined 55 studies, noted that "Although the causal relationship between IPV experience and alcohol consumption in women is far from clear, there is clear evidence that women with histories of violence consume more alcohol and, conversely, that women who binge drink and consume alcohol in other problematic ways are more likely to report experiences of violence" (p. 388) [18].

\section{Managing co-occurring IPV and mental health and substance use problems}

Although the evidence is irrefutable-women's experiences of IPV are connected in complex and reciprocal ways with poor mental health and substance use problemsresearch on the best treatment response for managing these co-occurring problems is both limited and more equivocal.

Interventions for women with co-occurring IPV and mental health problems have largely focused on studies of one type of counselling intervention, counselling for specific populations [26], or comparisons of two different counselling modalities [27]. Feder and colleagues [28] conducted a systematic review of 23 studies of psychological interventions for abused women with mental health problems who had left the abusive relationship. Individual and group interventions including expressive writing, forgiveness therapy, crisis intervention, and cognitive behavioral therapy were reviewed and outcomes compared. Although outcomes differed across studies, to varying degrees all interventions were somewhat helpful in ameliorating negative mental health symptoms (particularly depression and PTSD), although the improvements were not all statistically significant and in some cases improvements were noted in both the intervention and control groups. The authors concluded that there was some evidence for the efficacy of individual cognitivebased therapy for women experiencing PTSD who were no longer in the abusive relationship but not enough to endorse group psychological approaches. We know of no other recent systematic review or meta-analyses describing useful interventions for women experiencing co-occurring IPV and mental health problems.

Research on the management of co-occurring IPV and substance use problems is still in a nascent stage. Weaver and colleagues [29] examined select research on interventions for substance-using women who also experience IPV and found that "effective screening, brief interventions, co-ordinated systems of care, and treatment approaches to address these co-occurring problems remain very limited" (p. 51). Focusing on activities within domestic violence shelters, Schumacher and Holt [30] recently completed a review to consider the substance abuse treatment options and needs of shelter residents. They found no randomized controlled trials that examined the benefits of substance abuse treatment offered within domestic violence shelters. However, there was promising preliminary evidence that reductions in episodes of IPV and substance use followed women's participation in parallel or integrated treatment programs. Unfortunately, it was also noted that few shelters offered any onsite substance abuse treatment programs, although this may not be surprising given that many shelters have rules against the provision of services to substance-using abused women [31].

The challenges of integrating IPV into substance abuse treatment settings (and vice versa) may derive from the sectors' different philosophical orientations, ideologies, 
service delivery models, funding mechanisms, and desired outcomes [31]. Successfully overcoming these challenges was the focus of a review of collaborations negotiated between domestic violence and substance abuse services completed by Macy and Goodbourn [32]. Two different models of collaboration were identified. The first, co-ordinated, service delivery involved the two services working in close relationship with cross-training of providers, cross-screening of clients, and cross-referrals. The second model focused on the development of integrated care where both IPV and addiction-related services are provided in the same center. This model requires additional training in IPV for addiction workers. The authors concluded that, to ensure success, both models require "multidimensional strategies at various levels, including the provider, director, agency, and policy levels" ([32] p. 246).

A scoping review of studies published between 2005 and 2014 used IPV, mental health and/or substance use along with treatment, training, and education as key words [33]. While 35 articles were retrieved, just 14 examined concurrent IPV, mental health problems, and substance use/misuse. Nine of these were reports from a US-funded initiative, the Women Co-Occurring Disorders and Violence study, designed to support the implementation and evaluation of services for women with these co-occurring problems. Women in the intervention sites showed some improvements in mental health and substance-use related problems, although in some studies positive outcomes were shown for those in the various interventions as well as control groups. As the 14 sites (9 completed evaluations) implemented or integrated services in different ways, it was difficult to determine what contributed overall to success. Across the various publications described in this study, the key recommendation was the importance of developing integrated, trauma-informed care for women with cooccurring IPV and mental health and substance use. The specific nature and content of trauma-informed care was left unclear.

The need for guidelines on the management of cooccurring problems led the American Substance Abuse and Mental Health Services Administration (SAMHSA) to develop a series of Treatment Improvement Protocols (TIPs) to provide best practice guidance to clinicians, program administrators, and funders. For example, TIP number 25, Substance Abuse Treatment and Domestic Violence [34], provides recommendations such as the following: develop safety and relapse prevention plans for abused women entering substance abuse treatment; offer same-sex treatment groups that do not use confrontational techniques; have abused women sign a "no-contact contract" agreeing not to communicate with their abusive partner while in treatment; refer (as appropriate) for psychotherapy and specialized counseling; and provide staff training on crisis management. Although these appear to be sound recommendations, Brackley and colleagues [35] have reported that few substance abuse treatment settings have incorporated any of the recommendations from TIP 25 into practice.

\section{Conclusion}

Considering how common, complex, and mutually reinforcing the problems of mental health and substance misuse are for women who experience IPV, it is surprising how little is known about these issues and their effective management. To clarify the temporal nature of the relationships and improve services for these women, additional longitudinal studies using consistent measures and definitions are required. Nevertheless, there are some general principles that may prove useful while waiting for the evidence base to further develop. For example, although limited to IPV survivors' experiences of the mental health care system, a recent meta-synthesis of 12 qualitative studies completed by Trevillion and colleagues [36] offers insights into IPV survivors' wishes regarding their healthcare. Across the studies, survivors spoke of the importance of having professionals trained to identify and acknowledge the abuse, the necessity of safety being recognized as a priority issue, and were critical of the biomedical model of mental illness with its emphasis on diagnosing and treating illness without exploring underlying contributing factors. They also emphasized the need for better communication and co-ordination across services. To this list, we would add that it is important to keep the individual woman at the center of care, considering her specific needs when designing treatment plans or making referrals.

\section{Abbreviations}

IPV, intimate partner violence; PTSD, post-traumatic stress disorder; TIP, treatment improvement protocol.

\section{Disclosures}

The authors declare that they have no disclosures.

\section{References}

I. Heise L, Garcia-Moreno C: Violence by intimate partners. In World health report on violence and health. Edited by Krug E, Dahlberg LL, Mercy JA, et al. Geneva, Switzerland: World Health Organization. 2002; 87-I22. [http://whqlibdoc.who.int/hq/2002/924I5456I5.pdf]

2. Jewkes R, Sen P, Garcia-Moreno C: Sexual violence. In World health report on violence and health. Edited by Krug E., Dahlberg LL, Mercy JA, et al. Geneva, Switzerland: World Health Organization. 2002; 147-82. [http://whqlibdoc.who.int/hq/2002/924I5456 I5.pdf]

3. Violence against women: a 'global health problem of epidemic proportions' [News release]. World Health Organization 
(WHO); 20I3. [http://www.who.int/mediacentre/news/releases/20I3/ violence_against_women_20I30620/en/]

4. Devries KM, Mak, JYT, García-Moreno C, Petzold M, Child JC, Falder G, Lim S, Bacchus LJ, Engell RE, Rosenfeld L, Pallitto C, Vos T, Abrahams N, Watts $\mathrm{CH}$ : Global health. The global prevalence of intimate partner violence against women. Science (New York, N.Y.) $2013,340: 1527-8$.

5. Global and regional estimates of violence against women: prevalence and health effects of intimate partner violence and non-partner sexual violence. Geneva, Switzerland: World Health Organization. [http://apps.who.int/iris/bitstream//0665/85239/I/ 978924I564625_eng.pdf]

6. Stöckl H, Devries K, Rotstein A, Abrahams N, Campbell J, Watts C, Moreno CG: The global prevalence of intimate partner homicide: a systematic review. Lancet 2013, 382:859-65.

\section{FlOOOPrime
RECOMMENDED}

7. Siemieniuk, Reed AC, Krentz HB, Gill MJ: Intimate partner violence and HIV: a review. Current HIVIAIDS reports 2013, 10:380-9.

8. Dillon G, Hussain R, Loxton D, Rahman S: Mental and Physical Health and Intimate Partner Violence against Women: A Review of the Literature. International journal of family medicine 2013, 2013:313909.

FlOOOPrime RECOMMENDED

9. Ellsberg $\mathrm{M}$, Emmelin M: Intimate partner violence and mental health. Global health action 20|4, 7:25658.

10. Lagdon S, Armour C, Stringer M: Adult experience of mental health outcomes as a result of intimate partner violence victimisation: a systematic review. European journal of psychotraumatology 2014, 5.

\section{FlOOOPrime \\ RECOMMENDED}

I I. Beydoun HA, Beydoun MA, Kaufman JS, Lo B, Zonderman AB: Intimate partner violence against adult women and its association with major depressive disorder, depressive symptoms and postpartum depression: a systematic review and meta-analysis. Social science \& medicine (1982) 2012, 75:959-75.

\section{FlOOOPrime}

12. Kraanen FL, Vedel E, Scholing A, Emmelkamp, Paul MG: Prediction of intimate partner violence by type of substance use disorder. Journal of substance abuse treatment 2014, 46:532-9.

\section{FlOOPrime} RECOMMENDED

13. Schneider R, Burnette ML, Ilgen MA, Timko C: Prevalence and correlates of intimate partner violence victimization among men and women entering substance use disorder treatment. Violence and victims 2009, 24:744-56.

14. Smith PH, Homish GG, Leonard KE, Cornelius JR: Intimate partner violence and specific substance use disorders: findings from the National Epidemiologic Survey on Alcohol and Related Conditions. Psychology of addictive behaviors: journal of the Society of Psychologists in Addictive Behaviors 2012, 26:236-45.

\section{FlOOOPrime}

RECOMMENDED

15. Howard LM, Trevillion K, Khalifeh H, Woodall A, Agnew-Davies R, Feder G: Domestic violence and severe psychiatric disorders: prevalence and interventions. Psychological medicine 2010, 40:881-93.

16. Simonelli A, Pasquali CE, Palo F de: Intimate partner violence and drug-addicted women: from explicative models to genderoriented treatments. European journal of psychotraumatology 2014, 5.
17. Trevillion K, Oram S, Feder G, Howard LM: Experiences of domestic violence and mental disorders: a systematic review and meta-analysis. PloS one 2012, 7:e51740.

\section{FlOOPrime}

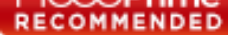

18. Devries KM, Child JC, Bacchus LJ, Mak J, Falder G, Graham K Watts C, Heise L: Intimate partner violence victimization and alcohol consumption in women: a systematic review and meta-analysis. Addiction (Abingdon, England) 2014, 109:379-91.

\section{FIOOOPrime
RECOMMENDED}

19. Devries KM, MakJM, Bacchus LJ, Child JC, Falder G, Petzold M, AstburyJ, Watts $\mathrm{CH}$, Tsai AC: Intimate Partner Violence and Incident Depressive Symptoms and Suicide Attempts: A Systematic Review of Longitudinal Studies. PLoS Med 2013, I0:e I001439.

20. Bundock L, Howard LM, Trevillion K, Malcolm E, Feder G, Oram S: Prevalence and risk of experiences of intimate partner violence among people with eating disorders: a systematic review. Journal of psychiatric research 2013, 47: I I34-42.

2I. Temple JR, Stuart GL, O'Farrell TJ: Prevention of intimate partner violence in substance-using populations. Substance use \& misuse 2009, 44:1318-28.

22. Gilbert L, El-Bassel N, Chang M, Wu E, Roy L: Substance use and partner violence among urban women seeking emergency care. Psychology of addictive behaviors: journal of the Society of Psychologists in Addictive Behaviors 2012, 26:226-35.

\section{FlOOOPrime \\ RECOMMENDED}

23. Afifi TO, Henriksen CA, Asmundson, Gordon JG, Sareen J: Victimization and perpetration of intimate partner violence and substance use disorders in a nationally representative sample. The Journal of nervous and mental disease 2012, 200:684-9I.

24. Boden JM, Fergusson DM, Horwood LJ: Alcohol misuse and violent behavior: findings from a 30 -year longitudinal study. Drug and alcohol dependence 20I2, I 22:135-4I.

25. Waller MW, Iritani BJ, Christ SL, Tucker Halpern C, Moracco KE, Flewelling RL: Perpetration of intimate partner violence by young adult males: the association with alcohol outlet density and drinking behavior. Health \& place 2013, 21:10-9.

26. Nicolaidis C, Mejia A, Perez M, Alvarado A, Celaya-Alston $R$ Quintero Y, Aguillon R: Proyecto Interconexiones: a pilot test of a community-based depression care program for Latina violence survivors. Progress in community health partnerships: research, education, and action 2013, 7:395-40I.

27. Crespo M, Arinero $M$ : Assessment of the efficacy of a psychological treatment for women victims of violence by their intimate male partner. The Spanish journal of psychology 2010, 13:849-63.

28. Feder G, Ramsay J, Dunne D, Rose M, Arsene C, Norman R, Kuntze S, Spencer A, Bacchus L, Hague G, Warburton A, Taket A: How far does screening women for domestic (partner) violence in different health-care settings meet criteria for a screening programme? Systematic reviews of nine UK National Screening Committee criteria. Health technology assessment (Winchester, England) 2009, I3:iii-iv, xi-xiii, I-I I3, I37-347.

29. Weaver TL, Gilbert L, El-Bassel N, Resnick HS, Noursi S: Identifying and intervening with substance-using women exposed to intimate partner violence: phenomenology, comorbidities, and integrated approaches within primary care and other agency settings. Journal of women's health (2002) 20I5, 24:5I-6.

\section{FIOOPrime \\ RECOMMENDED}

30. Schumacher JA, Holt, DJ: Domestic violence shelter residents' substance abuse treatment needs and options. Aggression and Violent Behavior (20I2). I7(3): I88-97.

3I. Mason R, Toner B: (20I2). Making connections: when domestic violence, mental health and substance use problems co-occur. Toronto, ON: Women's College Hospital. 
32. Macy RJ, Goodbourn M: Promoting successful collaborations between domestic violence and substance abuse treatment service sectors: a review of the literature. Trauma, violence \& abuse 20I2, | 3:234-5I.

FlOOPrime

RECOMMENDED

33. Mason R, O'Rinn SE: Co-occurring intimate partner violence, mental health, and substance use problems: a scoping review. Global health action 2014, 7:248I5.

34. Center for Substance Abuse Treatment. (1997). Substance abuse treatment and domestic violence. Treatment Improvement Protocol (TIP) Series, No. 25. HHS Publication No. (SMA) 12-4076. Rockville,
MD: Substance Abuse and Mental Health Services Administration. [http://www.ncbi.nlm.nih.gov/books/NBK64437/pdf/TOC.pdf]

35. Brackley MH, Williams GB, Wei CC: Substance abuse interface with intimate partner violence: what treatment programs need to know. The Nursing clinics of North America 2010, 45:58I-9, vi.

36. Trevillion K, Hughes B, Feder G, Borschmann R, Oram S, Howard LM: Disclosure of domestic violence in mental health settings: a qualitative meta-synthesis. International review of psychiatry (Abingdon, England) 20।4, 26:430-44.

FlOOOPrime

RECOMMENDED 